\title{
An Electron Microscope Study of Spore Structure and Development in Alternaria brassicicola
}

\author{
By R. CAMPBELL \\ Department of Botany, The University, Bristol
}

(Accepted for publication 30 July 1968)

\begin{abstract}
SUMMARY
Each cell of the mature spore of Alternaria brassicicola has a two-layered wall, the layer distant from the protoplast being melanized. The mature septa are five-layered, having two layers of secondary wall on either side of the septal partition which is itself three-layered. Each septum has one simple pore. New spores are produced by an outgrowth, through a pore, of the inner wall layer of the mother cell. Young spores have many small mitochondria and much vesicular endoplasmic reticulum; as they mature, lipid bodies and an unknown polyglucoside are produced. Mature spores have glycogen but very little if any lipid. The suggestion by other workers that vesicular endoplasmic reticulum, multivesicular bodies and lomasomes are involved in wall formation is supported.
\end{abstract}

\section{INTRODUCTION}

Alternaria brassicicola (Schw.) Wiltshire (= A. oleracea Milbraith) produces acropetal chains of dictyospores with melanized walls (Pl. I, fig. I). In the classification of Hughes (1953) the spores are porospores (his group VI), that is, spores that are produced by the extrusion of the protoplast through a pore in the conidiophore wall. Terminal cells of the spores produce new spores in a similar fashion, and side branches to the chain may form by the growth of functional conidiophores from the cells of the spores (P1. I, fig. I). The taxonomy, nutrition and light-microscope morphology have been summarized and extended by Joly (1964). He describes how the new spores are budded out from mother cells at the tips of the conidiophores or spores. He states that the protoplasm of the mother cell retracts and leaves the mother cell empty, thereby isolating the newly formed spore. If this is so, it is difficult to see how the nutrients for the development of the new spores are moved along the chain to the tip where the spores are developing. The electron microscope studies described in this paper clarify this.

The cytology of Alternaria species has been studied by Stall (1958) and Hartmann (I966), both of whom found that the cells of the vegetative mycelium and the spores are multinucleate. Hartmann (1966) also reported that all the nuclei in a spore chain are derived from one or a very small number of nuclei which migrate into the first spore budded from the conidiophore.

The electron microscopy of mature conidia of higher fungi is usually difficult because of the impermeability of the wall to fixatives and embedding materials. The ultrastructure of the conidia of several species has, however, been described (Fusarium by Acha, Aguirre, Uruburu \& Villanueva (1966), Marchant (1966 a, b); Botrytis by Buckley, 
Sjaholm \& Sommer (1966); Cochliobolus by Matsui, Nozu, Kikumoto \& Matsuura, (I962); Neurospora by Turian (I966) and his co-workers). Reviews of general fungal and spore ultrastructure have been published, such as those by Hawker (1965) and Bracker (1967). Melanized dictyospores and the formation of acropetal chains, the subjects of this study, have not been investigated at the ultrastructural level, possibly because of the difficulty of fixing and embedding such thick-walled spores, and the difficulty of preventing fragmentation of the chain.

\section{METHODS}

Alternaria brassicicola was selected because of its abundant sporulation and genetical stability in culture; the large size of the spores enabled them to be orientated for sectioning. The culture used was kindly supplied by Dr Q. Ruscoe of Exeter University.

The cultures were grown on potato glucose agar (Oxoid CM 139) at $25^{\circ}$ for 4 to Io days, depending on the degree of maturity that was required for the majority of the spores. A technique was developed to maintain chain continuity during fixation and embedding and to orientate the spores for sectioning: the results of this technique are assessed later. Pyramidal epon blocks were prepared with Beem (LKB Products Ltd.) capsules as moulds. The blocks were very thinly coated with Haupt's adhesive (Jensen, I962). The small square tops of the pyramids were then touched very lightly onto a colony of the fungus; some chains of spores (Pl. I, fig. I), and many individual ones, adhered to the surface. The block was quickly chilled on an ice-cold metal sheet to gel the adhesive and was then put into cold fixative. The fixative denatures the protein in the adhesive, preventing a later return to sol at room temperature.

Fixation was extremely difficult. Numerous attempts to use mixtures of glutaraldehyde, osmium tetroxide and acrolein produced poor results, but the following methods were satisfactory.

(I) Permanganate (modified from Luft (1956)). Unbuffered $2 \%(w / v)$ potassium permanganate (with one drop of $\mathrm{I} \%$ ( $\mathrm{v} / \mathrm{v})$ Brij (British Drug Houses Ltd.) bio-inert wetting agent $/ 200 \mathrm{ml}$.) was used at $4^{\circ}$ for $2 \mathrm{hr}$.

(2) Paraformaldehyde + glutaraldehyde + acrolein (modified from an unpublished method of Dr E. M. Rodriguez, Bristol University). Two g. paraformaldehyde were placed in $25 \mathrm{ml}$. of water at $90^{\circ}$ and $0.1 \mathrm{~N}$-sodium hydroxide added dropwise until the solution cleared; $25 \mathrm{ml}$. of this was mixed with $5 \mathrm{ml}$. of $25 \%(\mathrm{w} / \mathrm{v})$ glutaraldehyde and $0.6 \mathrm{ml}$. acrolein. This solution was diluted I: I with $0.2 \mathrm{M}$-phosphate buffer $(\mathrm{pH} 7 \cdot 4)$. The material was fixed for $6 \mathrm{hr}$, washed in $0 . \mathrm{I} \mathrm{M}$-buffer, and then fixed in $\mathrm{I} \% \mathrm{(w/v})$ osmic acid in $0.1 \mathrm{M}$-buffer for $16 \mathrm{hr}$, all at $4^{\circ}$.

After fixation the material, still attached to the epon block, was stained in a saturated aqueous solution of uranyl acetate for $2 \mathrm{hr}$ at $4^{\circ}$, dehydrated at $4^{\circ}$ in an ethanol + water series followed by propylene oxide, placed in two changes of $50+50$ propylene oxide + epon (with accelerator) at room temperature for periods of $24 \mathrm{hr}$ each, transferred to fresh epon for $24 \mathrm{hr}$ with three changes, and finally placed in Beem capsules with fresh epon and polymerized for 3 days at $35^{\circ}$, I day at $45^{\circ}$, and 2 days at $60^{\circ}$. Pyramids were cut for sectioning to include selected spores or spore chains whose approximate maturity had been determined under the light microscope. Photomicrographs were made of the pyramids before sectioning, and these were used to identify particular spores under the electron microscope by their relative positions in the section. This 
greatly assisted in the determination of the relative spore age in electron micrographs. Sections were cut on an LKB ultratome, mounted on Formvar-coated carbon-stabilized copper grids, stained with alkaline lead citrate (Reynolds, 1963) and viewed with an AEI 6B electron microscope.

Some spores were prepared for the Stereoscan electron microscope by attaching them to the metal specimen-holders with Haupt's adhesive and coating them with gold + palladium after drying.

\section{RESULTS}

The sequence of events in the spore production and maturation of Alternaria brassicicola described below is compiled from many individual observations; to place them in the correct sequence was difficult. The method of preparation and embedding, involving the attachment of the spores to epon blocks, achieved two objects. First, the somewhat delicate chains were held together, though shrinkage during dehydration frequently separated the spores slightly. Secondly, the arrangement of the spores in a flat plane enabled them to be studied with the light microscope before sectioning, and made longitudinal sections possible. All the structures described have been seen after at least two different fixation treatments, unless otherwise stated. This, together with information from light microscopy and the relative spore age, makes it virtually certain that the described sequence is the correct one. In the following description the development of the spore is traced from its emergence until maturity. The process is considered in three parts: wall development; the formation of new spores; changes in the cytoplasmic membranes and organelles.

\section{Wall and septum formation and maturation}

Three main parts of the wall are distinguished: the primary wall, which is the original wall formed when the spore was budded out; the septal partition, which is threelayered and forms the main part of the septum; the secondary wall, which surrounds individual cells of the spore and may line either the primary wall or both the primary wall and the septal partition (Fig. I).

The wall of the young spore is quite thin and has a granular electron-dense substance concentrated in the outer region (Pl. I, fig. 2). It remains in this condition throughout the very rapid increase in size of the spore which takes place during the first hour or so of growth. As the rate of enlargement slows, the wall develops two illdefined layers (Pl. I, fig. 3), the outer one having a granular texture and darker colour probably because of the deposition of melanin and the inner one being electrontransparent. The outer layer (the primary spore wall) is derived from the original wall of the young spore (Pl. I, fig. 2; Pl. 3, fig. I7; Fig. I A), while the inner layer (the secondary wall) surrounds individual cells of the spore as they are delimited by septum formation (P1. I, fig. 3; Figs. I B, C). The septal partition abuts onto the primary wall when the septum is laid down before the secondary wall formation begins (P1. I, fig. 3 at A; Fig. I B), but when the septal partition is laid down after the secondary wall it will abut onto the latter (P1. I, fig. 4; Fig. I C). Longitudinal septal partitions abut onto previous septa (P1. I, fig. 3 at B; Fig. I D), giving the muriform condition. In a large muriform spore there may be a succession of walls formed over some days as the spore matures. The only method of determining the order in which the septa have been formed is to study the place where the septal partition abuts in relation to 
the other wall layers. The secondary walls are thinner over the newer septal partitions than over the older ones (Pl. I, fig. 3), and are thickest of all where they are laid directly on the primary wall.

The details of the septal partition formation are difficult to observe; because the process takes only a matter of minutes (light microscope observations) it is fixed in a very small number of spores. The septum is first apparent as an invagination of the

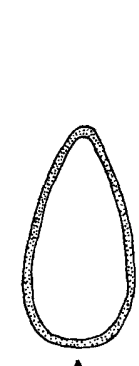

A

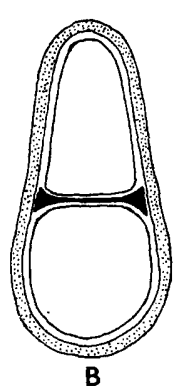

B

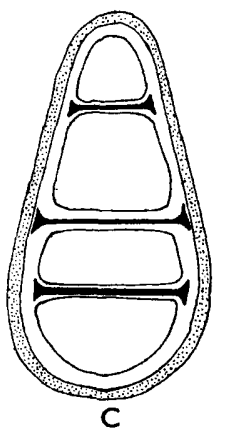

C

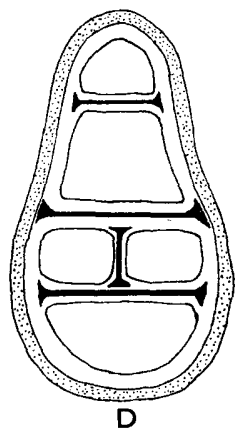

D

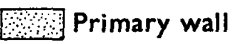

$\square$ Secondary wall

Septal partition

Fig. 1. Alternaria brassicicola. Diagram of wall layers in relation to septum formation (not to scale). A. The young spore having only the primary wall. B. The first septal partition is laid down abutting onto the primary wall, and the secondary wall is laid down in each cell. C. Further septal partitions are formed; the secondary wall has continued to grow in area and thickness and covers the septal partition. D. A mature spore in which a longitudinal septum has formed.

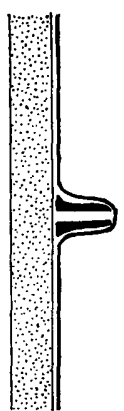

A

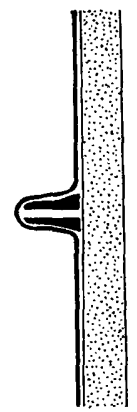

Primary wall

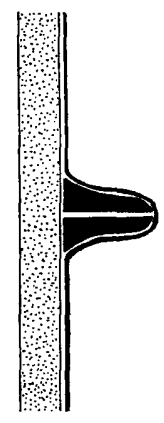

B

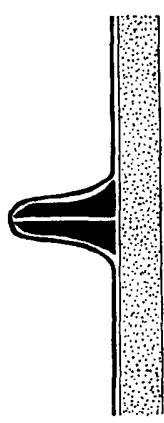

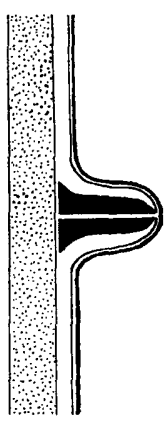

C

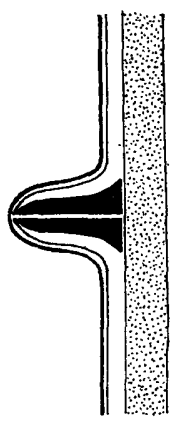

Septal partition $\quad$ Plasinalemma

Fig. 2. Alternaria brassicicola. Diagram of septum formation (not to scale; in median section). A. The plasmalemma invagination lays down the two layers of the septal partition, each in contact with the plasmalemma producing it and separated from each other by the electrontransparent layer. B. The invagination reaches the centre and presumably either leaves the pore open or fuses to give a complete septum in which the pore is subsequently formed. C. Secondary wall is laid over the septal partition so that each of the cells now formed, though in cytoplasmic continuity, has its own double wall of half the septal partition and a secondary wall. The walls of the two cells are separated by the electron-transparent layer. 
plasmalemma which develops on both sides of the spore in section and is presumably annular (Pl. I, fig. 5, 6; Fig. 2A). Within the invagination the septal partition is formed in two layers, each maintaining close contact with the membrane and separated from the other layer on the opposite side of the invagination by an electrontransparent layer (P1. I, fig. 6; Fig. 2A). The invagination grows towards the centre of the cell. The origin of septal pores, which are invariably present, has not been observed, but presumably either the closure of the invagination is incomplete or the pore is formed by perforation of the completed septal partition. In the young septal wall (P1. 2, fig. 7; Fig. 2 B) the electron-transparent dividing layer between the two layers of the septal partition is clearly seen. The secondary wall is laid down on this septal partition; what determines the change from one wall type to another is not clear. Each of the new cells is thus surrounded by its own double wall. The fully formed septum has five layers, these being the septal partition in which the two layers are separated by the electron-transparent layer and a layer of secondary wall which covers the partition on each side (P1. I, fig. 3; Fig. 2C).

The pore in the completed septum is simple (Pl. I, fig. 3, which is not quite median; Pl. 2, fig. 8). The septal partition and the secondary wall, when present, narrow abruptly and the plasmalemma passes around the lip where it is in direct contact with the layers of the septal partition. The pores are nearly always situated in the middle of the septum, and occur in longitudinal as well as transverse walls. In developing and mature spores the pores are invariably open; no pore plugs have been seen. Spherical membrane-bounded structures often occur near the pores (P1. I, fig. 3). These are usually electron-dense and are probably Woronin bodies (Bracker, 1967); sometimes, however, they appear electron-transparent, probably being poorly fixed and having lost their usual dense contents. Various organelles, including nuclei, have been seen passing through the pores.

Melanin deposition occurs from the time of spore formation, but is restricted, except in the very last stages of maturation, to the primary wall and septal partition of the spore, which become progressively more electron-dense (P1. 2, fig. 9) and finally completely opaque (compare Pl. I, fig. 2 to 4; Pl. 2, fig. 9). Very little, if any, melanin is laid down in any secondary wall even when it occurs between a septal and a primary wall (P1. 2, fig. I0). The electron-transparent layer which separates the two parts of the septal partition may become obscured by the melanin at maturity (P1. 2, fig. 9).

The rough and cracked appearance of the mature melanized wall ( $\mathrm{Pl}$. 2, fig. 9) is probably an artifact introduced during preparation and sectioning; it first becomes apparent when the spores are placed in propylene oxide. Optical and Stereoscan electron microscope photographs (P1. 2, fig. I I to I3) show smooth walls.

The spore walls are perforated by basal and apical pores which presumably maintain cytoplasmic continuity along the chain and allow transport of nutrients to the developing tip. The basal pore (P1. 2, figs. II, $\mathrm{I}_{3} ; \mathrm{Pl}$. 3, fig. I4, I5) is the connexion between the spore and its mother cell. The pore is usually a more or less cylindrical tube passing through the wall (P1. 3, fig. I4), but there may be a constriction at the boundary of the primary and secondary walls (Pl. 3, fig. I5). The apical pore is originally formed as a simple perforation of the primary wall through which the secondary wall blows out as a new bud (P1. 3, fig. 17). However, within an hour or two the wall around the pore starts to develop a ring of thickening, hereafter called the annulus, which lies within or just inside the secondary wall (P1. 3, fig. I6). 
This increases in size and becomes electron-dense, possibly due to the deposition of melanin, until it is clearly visible even with the light microscope (P1. 2, fig. I2). The function of this structure is unknown, but it is probably connected with mechanical support of the rest of the chain above the spore in question, the whole weight of which is borne by the very small area of contact around the pore. In the spores used in this study, which were mature but not dormant, the basal and apical pores were always open and cytoplasm could sometimes be seen 'leaking out' when the chain was broken during preparation (note, for example, the space left in Pl. 3, fig. 15). How, or whether, the pores are occluded in the dormant spore of a broken chain is not known; investigations are proceeding.

The basic wall structure is similar to that described for Botrytis (Buckley et al. 1966), though in Alternaria brassicicola the wall is much thicker and the structure and development much complicated by the presence of septa and the formation of a chain of spores. The multiseptate spore of Fusarium might be expected to be similar in some respects, but Marchant (1966 a, b) described the septa as completely continuous with the outside wall and similar to it in structure. This is not true of Alternaria, the wall of the latter being melanized, without the three layers described for Fusarium, and the spore having a much more complicated septum structure. A. brassicicola also has different septal partitions from Ascodesmis (Moore, 1963) which are not obviously composed of two dark layers separated by an electron-transparent one, though there is a suggestion of such a structure in one of the photographs (fig. II C of Moore, 1963). Bracker \& Butler (1963) described a similar septal partition (in the hyphae of Rhizoctonia) to that reported here, but no secondary wall was laid down on the partition and the septal pore was of the dolipore type.

\section{The formation of spores}

Spores are formed by the growth of a bud through a fairly well defined pore, which is apparently produced enzymically, for there is no sign of mechanical rupture (P1. 3, fig. I7, I8). The mother spore has usually formed one or more septa and has a secondary wall before it produces a spore. The pore is produced in the primary wall and the secondary wall grows out through it to form the primary wall of the new spore (Pl. 3, fig. 17). As this matures and melanin is deposited, the distinction between the new spore primary and the mother spore primary walls becomes less clear ( $\mathrm{Pl} .3$, fig. I8), but the discontinuity is indicated on the left of $\mathrm{Pl}$. 3, fig. 18, and is also shown by the fact that spores break away from the chain cleanly, without a tear in the melanized wall (Pl. 3, fig. I5). The discontinuity of melanized walls was pointed out by Simmons (1967) who used the optical microscope, though he did not observe the secondary wall and reported complete discontinuity between the walls of the mother cell and the daughter spore. The young spore rapidly expands, laying down the secondary wall and the septa as described above.

Spores are also produced from conidiophores; either from the original one, attached to the mycelium, which gave rise to the first spore in the chain, or, alternatively, after the enlargement of the spore has stopped, a growing point may appear in one of the lateral cells. A short conidiophore is rapidly produced (in about $10 \mathrm{~min}$., from optical microscope observations) and is usually walled off from the rest of the spore, apparently by a normal septum (P1. I, fig. I ; P1. 3, fig. I9). The apical cell may also produce a conidiophore rather than a spore direct. What determines this, and why a lateral cell 
develops a new growing point, is unknown. The formation of the conidiophore has not been seen on the electron microscope, but optical microscope observations suggest that growth takes place over a wide region of the spore wall rather than through a pore as in spore production. On some of the electron micrographs of mature conidiophores there is a suggestion of a collar of primary wall around the base (Pl. 3, fig. I9), indicating that the secondary wall may have mechanically broken and grown through the primary wall to form the conidiophore.

The conidiophore, which has both primary and secondary walls (P1. 3, fig. 19, 20), produces a new spore at its apex in the same way as described above for spores, and an annulus forms around the pore.

The cytoplasm of the conidiophore is sometimes very sparse and apparently degenerate ( $\mathrm{Pl}$. 3, fig. 20). This probably accounts for the reports from light-microscope work (Joly, 1964) that the tips of the conidiophores and spores were empty.

\section{Cytoplasmic maturation}

Cytoplasmic maturation is a complex process because of the large numbers of organelles and membrane systems involved and will for convenience be divided into stages related to the wall maturation. It is, however, a continuous process. The very young spore (Pl. I, fig. 2) has many small, irregularly shaped mitochondria, and much endoplasmic reticulum which is often vesicular. The plasmalemma is convoluted and associated with small vesicles (P1. 4, fig. 28); these are thought to be connected with the wall formation and will be discussed later when considering the maturing spore, in which they are more prominent. One or two nuclei are always present and their double membranes show well-defined pores. The presence of many mitochondria and extensive endoplasmic reticulum is compatible with a rapidly metabolizing and growing cell.

The cytoplasm of the maturing spore (Pl. I, fig. 3; Pl. 4, fig. 2I) also has plentiful endoplasmic reticulum and mitochondria which are possibly somewhat larger than in the young spore. The main difference is the presence of lipid bodies and some vacuoles. An unknown substance is also present: it has an amorphous structure and appears as electron-transparent aggregates usually located around the edges of the protoplast (Pl. I, fig. 3; P1. 4, fig. 22). Preliminary chromatography of its hydrolysis products indicates that it is a polymer of glucose. It gives none of the usual cytochemical reactions of starch or glycogen and its exact composition is unknown, though some of its staining properties, particularly with toluidine blue (Pearse, I953), suggest that it may be a dextran. The structure shown on the photographs suggests that the substance has probably been removed during fixation and/or dehydration.

The lipid bodies have the usual structure (Pl. I, fig. 3, Pl. 4, fig. 2I), and have also been shown to be present by standard Sudan and osmium-staining methods for optical microscopy. There are also structures that are thought to contain phospholipid (Pl. 4, fig. 23, 24); they are very similar to those described by Buckley et al. (I966). Smith \& Marchant (1968) reported similar bodies in yeast and concluded that they were spherosomes whose phospholipid was used to form myelin bodies in vacuoles.

The vacuoles are for the most part empty (Pl. I, fig. 3). Some of them occasionally have multiple membranes around them (Pl. 4, fig. 25) or membrane complexes within them (Pl. 4, fig. 26). The membranes are too widely spaced for true myelin bodies; Smith \& Marchant (1968) pointed out that these loose aggregates of membranes, 
which have been reported in various fungi, should not be confused with true myelin bodies. It is noticeable that in Alternaria brassicicola they are seen only in material fixed with formaldehyde+glutaraldehyde + acrolein; this suggests that they may be an artifact of this particular method of fixation.

There are also complex membrane structures associated with the convoluted plasmalemma of maturing spores, which together with the multivesicular bodies and endoplasmic vesicles in the cytoplasm, are thought to be concerned with extensive secondary wall formation which is occurring at this time. This role in wall formation was proposed by Marchant, Peat \& Banbury (1967); they postulated that vesicles and multivesicular bodies were produced by the endoplasmic reticulum and then fused with the plasmalemma, discharging their contents, possibly enzymes or wall material, onto the developing wall. Multivesicular bodies can be seen in the cytoplasm and also outside the plasmalemma (P1. 4, fig. 22, 27). Extensive endoplasmic reticulum in the region of the walls appears to be budding off small vesicles (Pl. I, fig. 2; Pl. 4, fig. 2I, 28) into the cytoplasm which fuse with the plasmalemma (Pl. I, fig. 2; Pl. 2, fig. 8; Pl. 4, fig. 2I, 28). There are more complex elaborations of membranes in the region of the plasmalemma; some of these appear to be the fusion of membrane-containing vacuoles (Pl. 4, fig. 29) and presumably the vacuolar contents pass into the wall. These have not been seen in permanganate-fixed material. Finally, there are complex lomasomes (P1. 4, fig. 30) very similar to those shown by Moore \& McAlear (196I, Fig. 8) in their original paper on these structures. Marchant et al. (1967) illustrated rather more organized bodies and referred to them as plasmalemma elaborations. The latter authors pointed out that all these vesicular systems, interpreted by them and by Moore \& McAlear (196I) as fusing with the plasmalemma and contributing to wall formation, might in fact be moving in the reverse direction and be pinocytotic vacuoles in the sorts of hyphae from which they were first described. However, Marchant et al. (1967) found them in aerial sporangiophores of Phycomyces, and they are here shown to be present in aerial chains of spores of Alternaria brassicicola, where pinocytosis could not occur. This supports the argument that they are in fact moving outwards and depositing material in the walls.

The cytoplasm of mature spores is very different from that of either the young or the maturing spore. There are few mitochondria and generally very little endoplasmic reticulum (Pl. 2, fig. 9). The plasmalemma is relatively smooth and the vesicular systems largely absent. This indicates a great decrease of the metabolic rate and a cessation of wall synthesis. There are a few small vacuoles, but these are not as prominent as they are in the maturing spore. Lipid is absent or rare and no phospholipid bodies have been seen in mature spores. The polyglucoside is present in small amounts. The only additional substance found in mature spores is glycogen, which is frequently abundant (Pl. 2, fig. 9; Pl. 4, fig. 3I). It appears as electron-dense composite particles ( $\alpha$ particles) randomly distributed in the cytoplasm.

These changes in lipid and polysaccharide content of the spore have been checked by light microscope observations with standard cytochemical techniques for the localization of the substances. The following methods were used: Sudan dyes and osmium tetroxide for lipids, periodic acid Schiff's reagent for insoluble carbohydrates, Bauer-Feulgen and Best's carmine for glycogen, toluidine blue for the dextran-like substance (Glick, 1949; Jensen, 1962; Pearse, 1953).

Permanganate fixation does not preserve ribosomes, and the other fixatives used 
did not generally give good enough results of all stages in the maturation for the changes in ribosome distribution to be found. Optical microscope stains for RNA showed the greatest density in young and maturing spores, with practically none in the mature ones.

\section{DISCUSSION}

The study of chains of spores of Alternaria brassicicola has allowed detailed observations on maturation. Changes in the cytoplasmic structures follow a readily interpretable pattern, linked with the rate of metabolism, that would be expected in the various ages of spore; changes in mitochondria and endoplasmic reticulum are particularly obvious. The significance of the shift in the energy reserves from lipid and a polyglucoside in maturing spores to glycogen and a polyglucoside in mature spores is unknown. The sequence of the wall structure changes is complex. Investigations on germinating spores and hyphae are being continued to complete the full cycle of wall production in $A$. brassicicola. The wall structure of the spores is unlike any previously described and the distinction between euseptate and distoseptate spores as proposed by Luttrell (1963) on the basis of light microscopy is difficult to make. In euseptate spores the cells remain together as a single unit; in distoseptate spores each individual cell is surrounded by its own wall and separates from the other cells of the spore when the enclosing spore envelope is broken. Alternaria brassicicola would seem, from optical microscopy, to be euseptate, but the electron microscopy shows that each cell is surrounded by its own wall. Furthermore, in a spontaneous albino mutant of this strain the spores seem to be distoseptate, for the individual cells may fall apart when the outer envelope ruptures (unpublished results). The multiseptate spore of Fusarium (Marchant, 1966a) is euseptate, for the septa are continuous with the outer wall and the spores show no tendency to disintegrate.

The mode of spore production by Alternaria brassicicola poses problems about the exact differences between the primary and secondary walls. The secondary wall of the mother cell gives rise to and is continuous with the primary one of the new spore, which in turn has a secondary wall lining it. Primary walls seem incapable of much further growth in thickness after they start to become melanized. Only the secondary or young primary walls, next to the cytoplasm, can grow in area or thickness, and this is probably linked with the deposition of new wall material by the plasmalemma and associated organelles. It would appear that the difference between the primary wall and septal partition and the secondary wall may be not so much in structure as in their position relative to the plasmalemma. It is not known how, or why, the deposition of melanin occurs only in the walls farthest away from the protoplast. Transport of materials up the chain to the developing spores at the tip must occur, and a welldeveloped system of pores is present which maintains cytoplasmic continuity along the chain. Even cells that are apparently empty when viewed with the optical microscope do in fact contain some cytoplasm and therefore maintain this continuity. This transport up the chain raises problems of compartmentalization within the chain, for the spores at the base are maturing while material is passing through them to the developing ones at the tip. How neighbouring spores maintain a different physiological state while in close cytoplasmic connexion is not understood. It is possible that chain length is determined by the maturation of spores at the base, growth stopping either when all the nutrients are removed from the translocation stream before it reaches the 
tip or when transport through the mature spores at the base is for some reason impossible. Both these possibilities would explain why spores at the tip of a mature chain are often smaller than those at the base; they have been cut off from the nutrient supply and have matured before reaching their full size. Similarly spores produced on a poor culture medium are smaller than those from a rich medium (Joly, I964). However, this does not imply that maturation is caused by lack of nutrients, for spores will mature at the base of a chain while new ones are still being produced at the tip.

Thanks are due to Dr M. F. Madelin for helpful discussion throughout this study.

\section{REFERENCES}

Acha, I. G., Aguirre, M. J. R., Uruburu, F. \& Villanueva, J. R. (1966). The fine structure of the Fusarium culmorum conidium. Trans. Br. mycol. Soc. 49, 695.

BraCKer, C. E. (1967). Ultrastructure of fungi. A. Rev. Phytopath. 5, 343.

BrACKer, C. E. \& BUTLER, E. E. (1963). The ultrastructure and development of septa in hyphae of Rhizoctonia solani. Mycologia 55, 35.

Buckiey, P. M., Sjaholm, V. E. \& Sommer, N. F. (I966). The electron microscopy of Botrytis cinerea conidia. J. Bact. $91,2037$.

GuICK, D. (1949). Techniques of Histo- and Cyto-chemistry. New York: Interscience Publ. Inc.

Hartmann, G. C. (1966). The cytology of Alternaria tenuis. Mycologia 58, 694.

HAWKER, L. E. (1965). Fine structure of fungi as revealed by electron microscopy. Biol. Rev. 40, 52.

Hughes, S. J. (1953). Conidiophores, conidia and classification. Can. J. Bot. 3I, 577.

JensEN, W. A. (1962). Botanical Histochemistry. San Francisco and London: W. H. Freeman.

JoLY, P. (1964). Le genre Alternaria. Paris: Editions Paul Chevalier.

LurT, J. H. (1956). Permanganate-a new fixative for electron microscopy. J. biophys. biochem. Cytol. 2, 789.

LutTRELL, E. S. (1963). Taxonomic criteria in Helminthosporium. Mycologia 55, 643.

MARChant, R. (1966a). Fine structure and spore germination in Fusarium culmorum. Ann. Bot., N.S. 30, 44I.

MarchaNT, R. (1966b). Wall structure and spore germination in Fusarium culmorum. Ann. Bot., N.S. 30, 821.

Marchant, R., Peat, A. \& Banbury, G. H. (1967). The ultrastructural basis of hyphal growth. New Phytol. 66, 623.

Matsui, C., Nozu, M., Kikumoto, T. \& MatsuURa, U. (1962). Electron microscopy of the conidial cell wall of Cochliobolus myobeanus. Phytopathology 52, 717.

MooRE, R. T. (1963). Fine structure of Mycota. I. Electron microscopy of the Discomycete Ascodemis. Nova Hedwigia 5, 263.

MOORE, R. T. \& MCAlear, J. H. (I96I). Fine structure of Mycota. 5. Lomasomes-previously uncharacterized hyphal structures. Mycologia 53, 194.

Pearse, A. G. E. (1953). Histochemistry Theoretical and Applied. London: J. and A. Churchill.

REYNoLDS, E. S. (1963). The use of lead citrate at high $\mathrm{pH}$ as an electron opaque stain in electron microscopy. J. Cell Biol. 17, 208.

Simmons, E. G. (1967). Typification of Alternaria, Stemphylium and Ulocladium. Mycologia 59, 67.

Smith, D. G. \& MARChant, R. (1968). Lipid inclusions in the vacuoles of Saccharomyces cerevisiae. Arch. Mikrobiol. 60, 340.

Stall, R. E. (1958). An investigation of nuclear number in Alternaria solani. Am. J. Bot. 45, 657.

TURIAN, G. (1966). The genesis of macroconidia of Neurospora. In The Fungus Spore. Colston Pap. 18,61 . 


\section{EXPLANATION OF PLATES}

Key to symbols used on plates: $P W=$ primary wall; $S W=$ secondary wall; $S P=$ septal partition; $\mathbf{P}=$ plasmalemma $; \mathbf{E R}=$ endoplasmic reticulum $\mathbf{N}=$ nucleus $; \mathbf{M}=$ mitochondrion $\mathbf{V}=$ vacuole; $\mathbf{L}=$ lipid body $; \mathbf{G}=$ glycogen $\mathbf{P G}=$ polyglucoside; $\mathbf{M C}=$ mother cell. Scale lines represent distances in microns $(\mu)$ as indicated.

\section{Plate I}

Fig. 1. Light microscope picture of a spore chain of Alternaria brassicicola. Note the branching chains, conidiophores on the spores and the very young spores at the chain tips. The scale line should read $20 \mu$ not $10 \mu$ as shown. $\times 600$.

Fig. 2. Young spore. There are numerous mitochondria and plentiful endoplasmic reticulum, but no vacuoles, lipid or storage products. $\mathrm{KMnO}_{4} . \times 7750$.

Fig. 3. Part of a medium-aged spore. The wall is two-layered and the septa have pores. Note the positioning of the ends of each septal partition in relation to the other wall layers. At A the septal partition abuts onto the primary wall and at $B$ it abuts onto another septum. The cytoplasm has vacuoles, lipid bodies and the unknown polyglucoside. Formaldehyde + glutaraldehyde + acrolein, postfixed in osmium tetroxide. $\times 7500$.

Fig. 4. The septal partition abuts onto the secondary wall, having been formed after it. $\mathrm{KMnO}_{4}$. $\times 30,000$.

Fig. 5. The start of septum formation, with the plasmalemma invaginating. $\mathrm{KMnO}_{4} \times \mathrm{II}_{1}, 00$.

Fig. 6. Detail of fig. 5. Note how the plasmalemma on each side of the invagination lays down its own layer of the septal partition, leaving an electron-transparent layer between the two dark layers of the partition. $\mathrm{KMnO}_{4} \cdot \times 25,000$.

\section{Plate 2}

Fig. 7. The young septal partition formed between the primary wall and another septum: the partition is composed of three layers, two granular ones separated by an electron transparent-one. The section is not quite median, hence no pore is visible. $\mathrm{KMnO}_{4}$. $\times 17,500$.

Fig. 8. Median section of a septal partition with a pore, before the secondary wall is laid down. The septal partition is divided by the electron-transparent layer and the plasmalemma is continuous over the lip. Note the large amounts of endoplasmic reticulum and vesicles formed from it. $\mathrm{KMnO}_{4}$. $\times 27,500$.

Fig. 9. A cell of a mature spore. The primary wall and septal partition are heavily impregnated with melanin. There are few vacuoles and mitochondria, little polyglucoside and no lipid in the cytoplasm, but glycogen is present. $\mathrm{KMnO}_{4} . \times 15,400$.

Fig. 10. Part of a mature spore showing an unmelanized secondary wall between the primary wall and the septal partition. $\mathrm{KMnO}_{4} \times \mathbf{2 4 , 3 7 5}$.

Fig. II. Light-microscope photograph of a spore with a two-layered wall and a basal pore where it was joined to the mother cell. $\times 2000$.

Fig. I2. As fig. II, but showing the apical pore with the annulus. $\times 2100$.

Fig. 13. Stereoscan electron microscope photograph showing the smooth wall and basal pore. $\times$ about 2350.

Plate 3

Fig. I4. Basal pore in the nearly mature spore: the secondary wall lines the pore. $\mathrm{KMnO}_{4} . \times 35,000$. Fig. 15. As fig. 14, but the pore has a constriction where it passes through the junction between the primary and secondary walls. $\mathrm{KMnO}_{4} . \times 26,250$.

Fig. 16. Apical pore of a spore with a well-developed annulus. $\mathrm{KMnO}_{4} \times 13,500$.

Fig. 17. A very young spore just being produced. The bud is surrounded by a wall which is continuous with the secondary wall of the mother cell. $\mathrm{KMnO}_{4} . \times 29,000$.

Fig. I8. A part of a more mature spore attached to its mother cell. There is continuity between the secondary wall of the mother cell and the new primary wall of the spore. $\mathrm{KMnO}_{4} \times 24,000$.

Fig. 19. A conidiophore produced by one of the lateral cells of a spore, forming a branching point in the chain. Formaldehyde + glutaraldehyde + acrolein; postfixed in osmium tetroxide. $\times 8000$.

Fig 20. An apical conidiophore, on a spore, whose contents are degenerating. The annulus has not been cut in median section. Formaldehyde + glutaraldehyde + acrolein; postfixed in osmium tetroxide. $\times \mathbf{I 5 , 0 0 0}$. 


\section{Plate 4}

Fig. 21. Cell of a medium-aged spore. The cytoplasm contains mitochondria and many lipid bodies, endoplasmic reticulum is plentiful and in many places seems to be budding off small vesicles. $\mathrm{KMnO}_{4}$. $\times 17,500$.

Fig. 22. Detail of the polyglucoside appearing as amorphous electron-transparent aggregates. $\mathrm{KMnO}_{4}$. $\times 50,000$.

Fig. 23, 24. Bodies thought to contain phospholipid. $23, \mathrm{KMnO}_{4} . \times 25,000 ; 24$, Formaldehyde + glutaraldehyde + acrolein; postfixed in osmium tetroxide. $\times 48,000$.

Figs. 25, 26. Vacuoles surrounded by or containing multiple membrane complexes. 25, $\times 50,000$; 26, $\times 58,000$. Formaldehyde + glutaraldehyde + acrolein; postfixed in osmium tetroxide.

Fig. 27. A lomasome in a medium-aged spore. Formaldehyde+glutaraldehyde+acrolein; postfixed in osmium tetroxide. $\times \mathbf{7 2 , 5 0 0}$.

Fig. 28. Detail of fig. 2. The endoplasmic reticulum has cysternae which are cut off to form vesicles which subsequently fuse with the plasmalemma. $\mathrm{KMnO}_{4} . \times 52,500$.

Fig. 29. A membrane complex associated with the plasmalemma. Formaldehyde + glutaraldehyde + acrolein; postfixed in osmium tetroxide. $\times 37,500$.

Fig. 30. A complex lomasome in a medium-aged spore. Formaldehyde + glutaraldehyde +acrolein; postfixed in osmium tetroxide. $\times 30,000$.

Fig. 31. Detail of fig. 9 showing abundant glycogen. $\mathrm{KMnO}_{4} . \times 30,000$. 
Journal of General Microbiology, Vol. 54, No. 3

Plate I

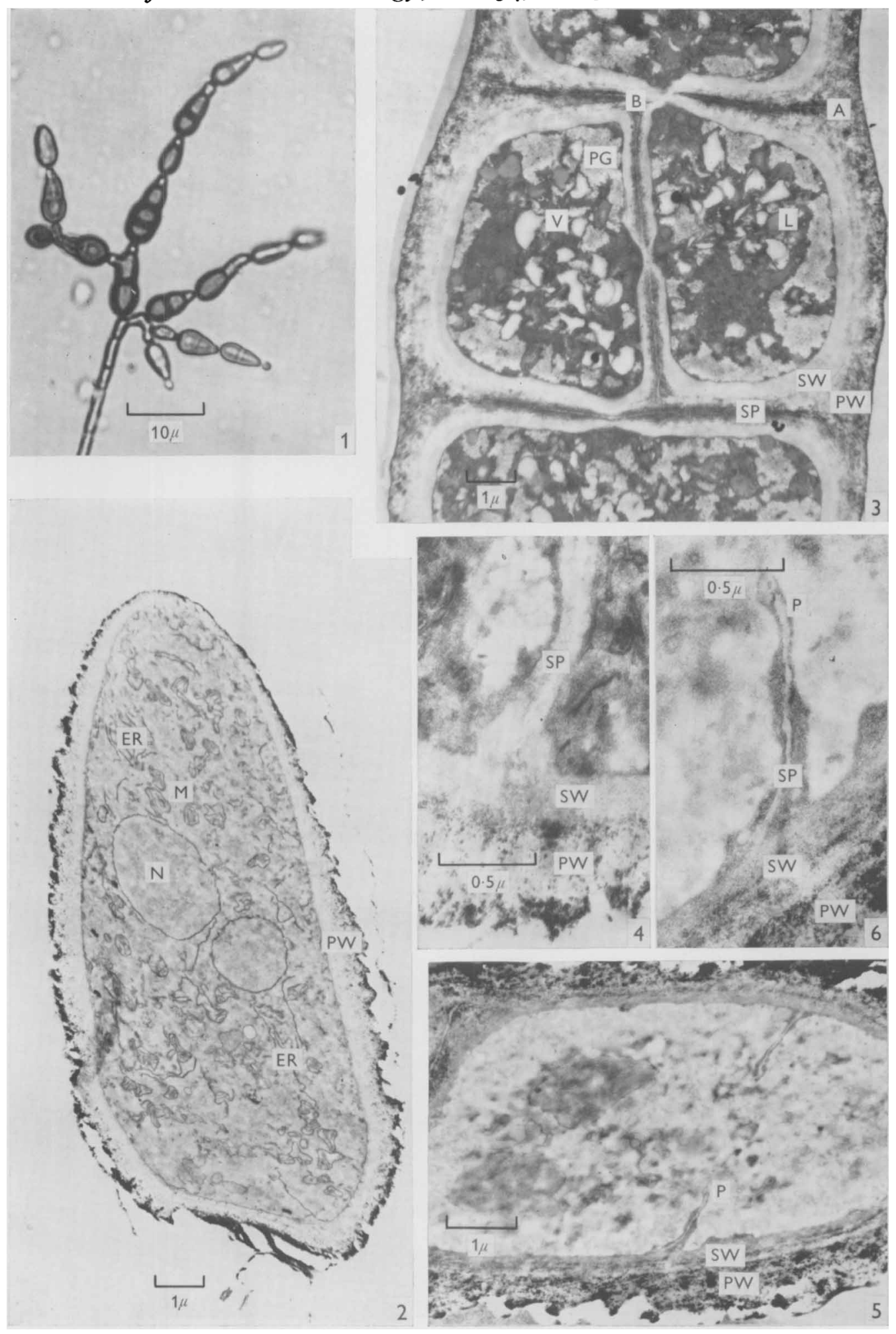

R. CAMPBELL

(Facing p. 392) 
Journal of General Microbiology, Vol. 54, No. 3

Plate 2
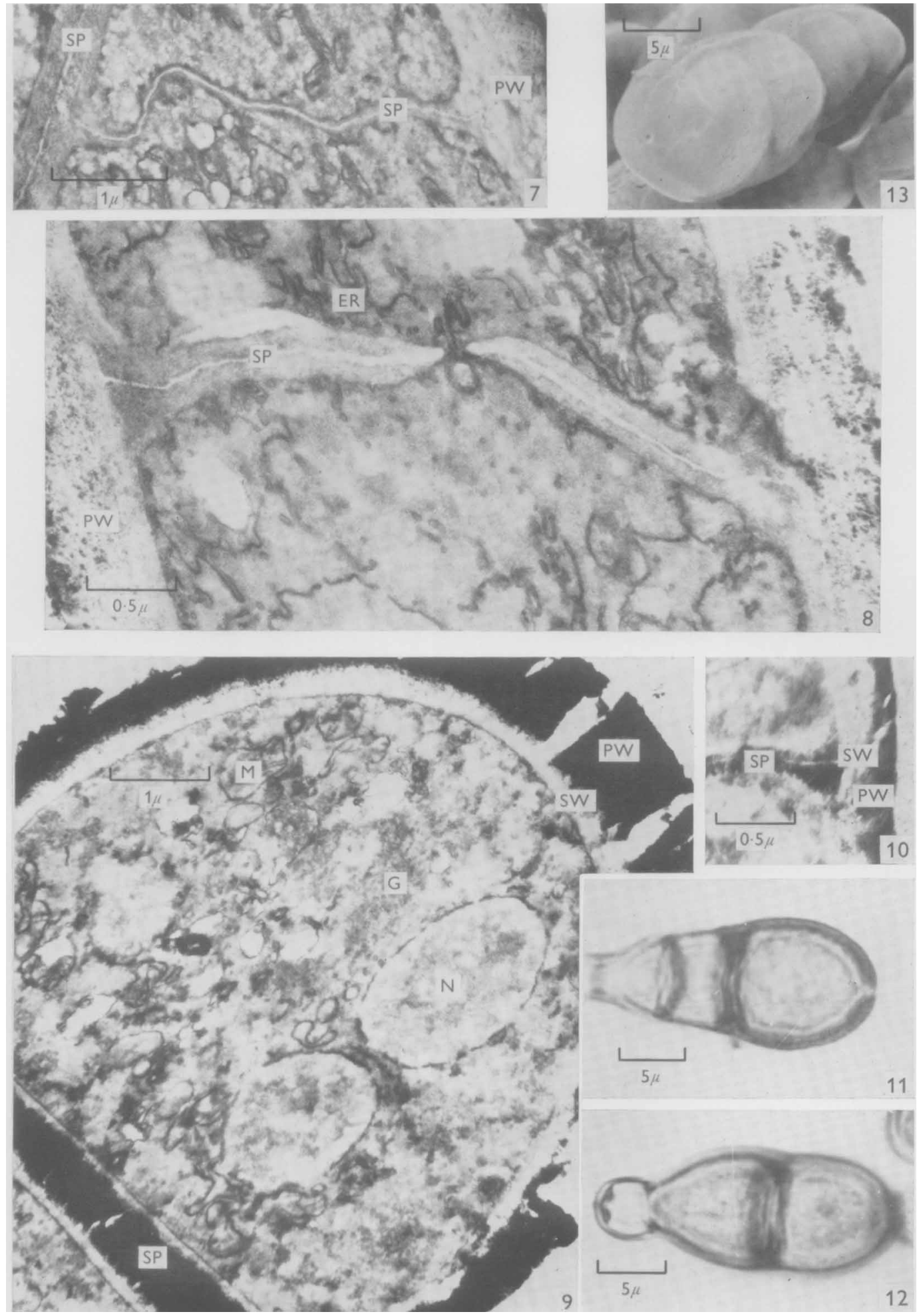

R. CAMPBELL 
Journal of General Microbiology, Vol. 54, No. 3

Plate 3

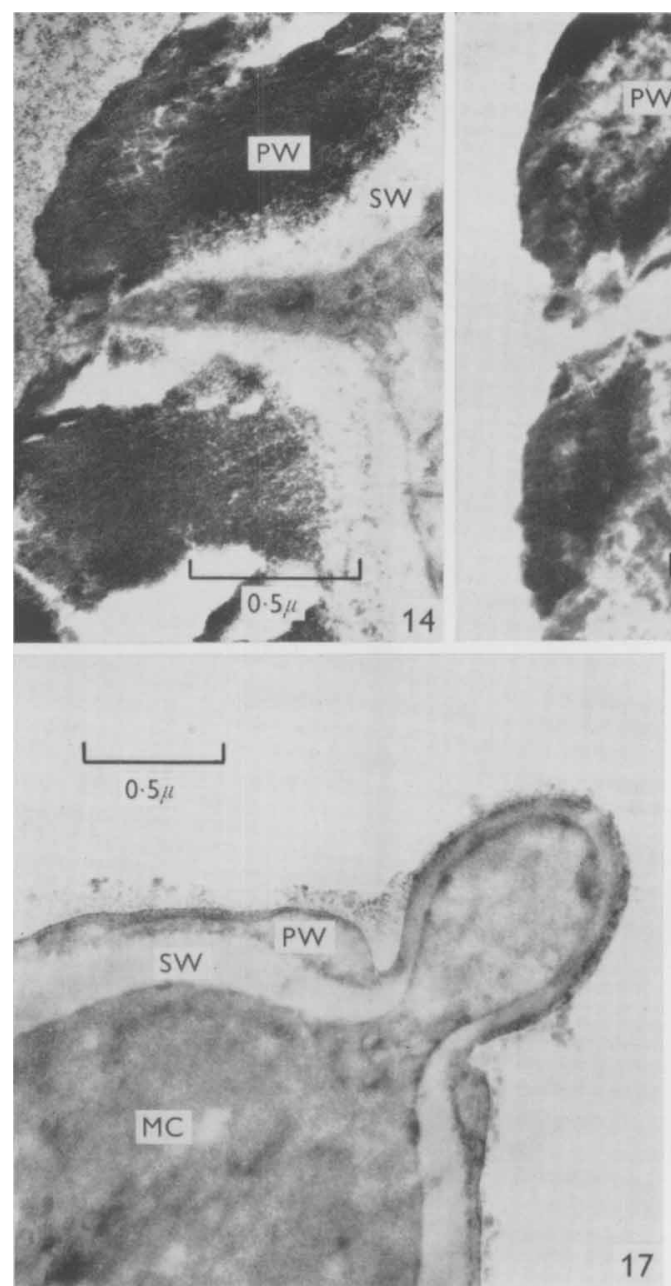

17
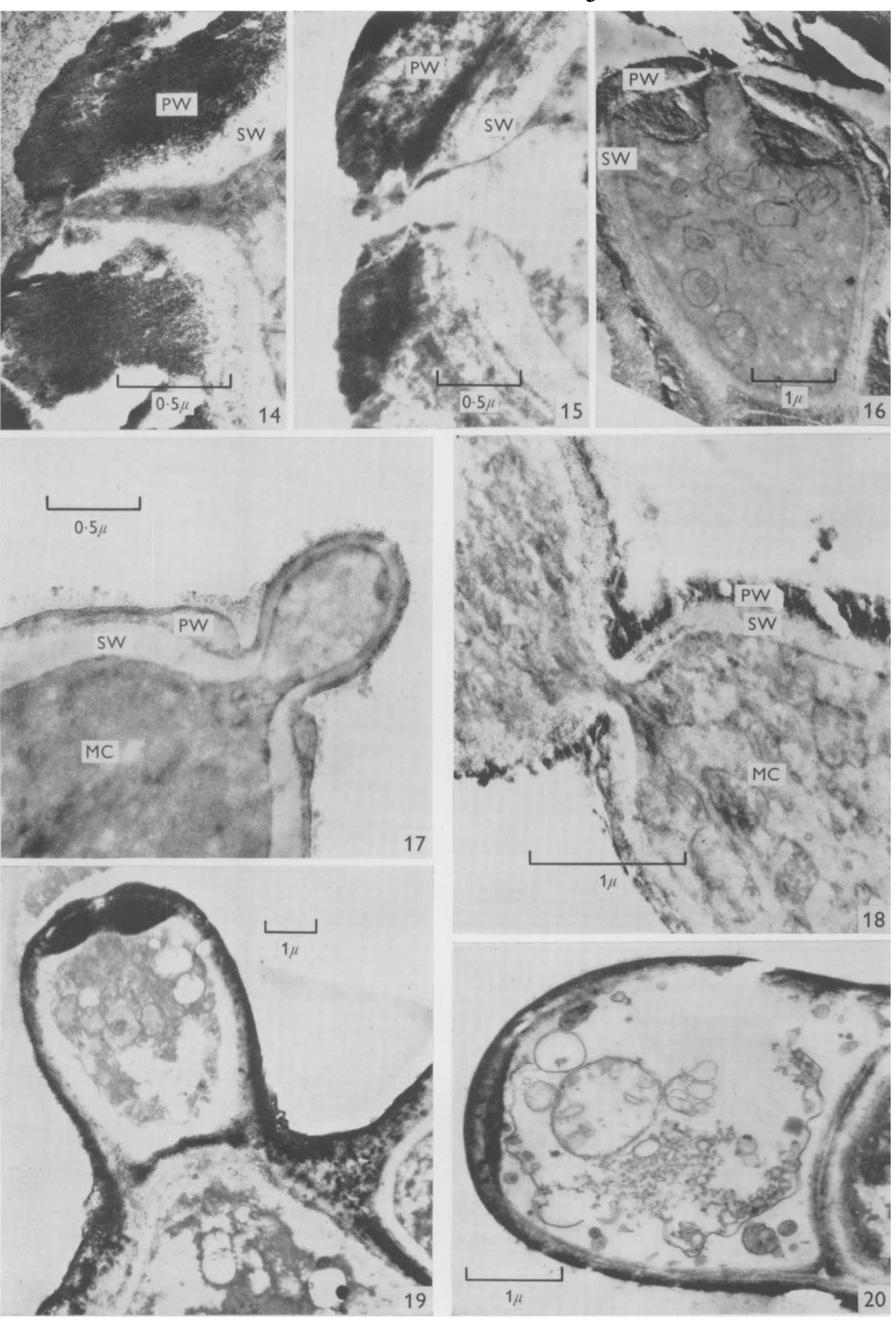

R. CAMPBELL 
Journal of General Microbiology, Vol. 54, No. 3

Plate 4

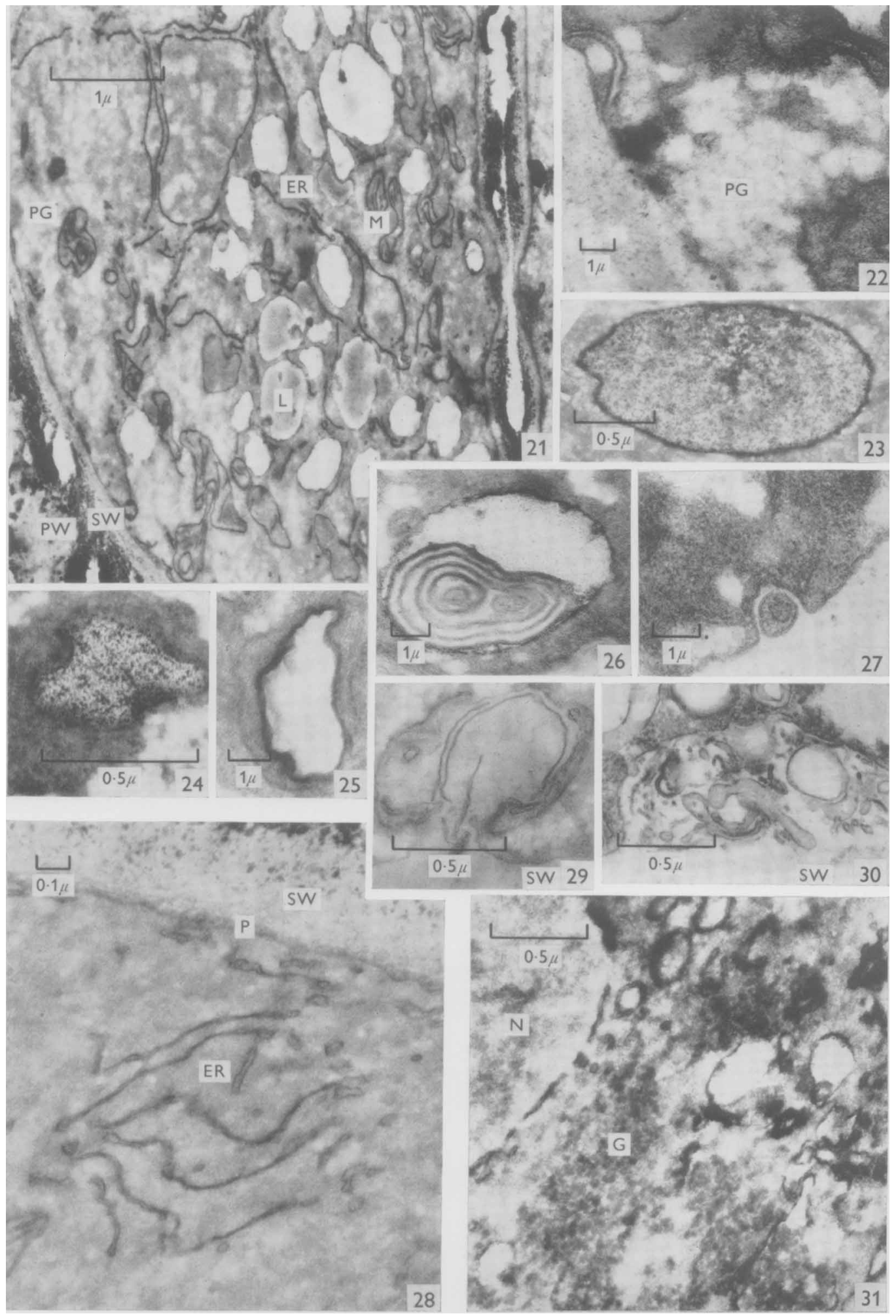

R. CAMPBELL 